\title{
Risk Factors for Mortality in Pediatric Cardiac Intensive Care Unit
}

\author{
Cristiane Martins ${ }^{1 \oplus}$ and Bruna M. N. Gama ${ }^{1,2}$ \\ Biocor - Hospital de Doenças Cardiovasculares, ${ }^{2}$ Nova Lima, MG - Brazil \\ Faculdade de Medicina de Barbacena, ${ }^{2}$ Barbacena, MG - Brazil. \\ Editorial related to the article: Clinical and Epidemiological Profiles of Patients Admitted to a Pediatric Cardiac Intensive Care Unit
}

Congenital heart defect commonly requires care in pediatric intensive care unit (PICU). They are a heterogeneous group of disorders with an annual incidence of 25,757 new cases in Brazil, 12 cases per 1,000 inhabitants $^{1}$. In this paper, Guimarães et al., ${ }^{2}$ published a cross-sectional study to describe the epidemiology of patients admitted to PICU from a tertiary hospital in Brazil. In this editorial, we review recent progress in understanding the risk factors for mortality in PICU. The editorial was produced by searching Pubmed and Scielo, using the terms "PICU", "RACHS", "CHD", and "mortality".

Congenital heart defects (CHD) are serious and common conditions that have a significant impact on morbidity, mortality and healthcare costs in both children and adults. ${ }^{3}$ It is estimated that at least 32,000 infants in the United States will be affected each year by CHD. ${ }^{3}$ Of these, approximately $25 \%$, or 2.4 per 1,000 live births require invasive treatment in the first year of life. While advances in treatment in the last decades have decreased infant mortality, they have also led to an increase in the number of children and adults with CHD. ${ }^{4}$ Despite these advances and developments in interventional and surgical techniques, heart disease in children remains an important cause of morbidity and mortality. ${ }^{5}$

Although acquired defects contribute to hospitalizations in PICU, congenital diseases are more prevalent. In developed countries, Kawasaki disease is the main cause of acquired heart disease in children younger

\section{Keywords}

Heart Defects Congenital/mortality; Risk Factors; Mordity; Intensive Care Units; Hospitalization; Heart Septal Defects, Ventricular; Aortic Coarctation; Arterial Switch Operation.

\section{Mailing Address: Cristiane Martins}

Biocor Hospital de Doenças Cardiovasculares Ltda - Cardiologia Pediátrica Alameda Oscar Niemeyer, 217. Postal Code: 34000-000, Nova Lima, MG - Brazil.

E-mail: cristianemar@hotmail.com than five years old. In underdeveloped and developing countries, such as Brazil, rheumatic carditis is the main cause of acquired heart disease. In the series Guimarães et al., ${ }^{2}$ acquired heart disease accounted for $8.1 \%$, with rheumatic heart disease being the main cause. The most common diagnoses of acyanotic heart defects were interventricular communication ( $24.5 \%)$, followed by total atrioventricular septal defect (19.9\%), persistent arterial channel and interatrial communication (13.2\% each) and coarctation of the aorta $(11.9 \%)$. Among the cyanotic congenital heart defects, the most common was tetralogy of Fallot $(30 \%)$, followed by tricuspid atresia (17.6\%), complex heart diseases $(15.3 \%)$, pulmonary atresia $(9.9 \%)$, transposition of the great arteries $(9.2 \%)$, complex heart diseases with PS (6.1\%), total anomalous pulmonary venous return $(6.1 \%)$, single ventricle and truncus arteriosus (2.3\% each) and Einstein anomaly $(0.8 \%)$. These data coincide with those published by several authors. Like other authors, Guimaraes, et al., ${ }^{2}$ used the risk stratification of patients admitted to the PICU: the RACHS score. The RACHS-1 score is a simple model that can be easily applied because it requires little data. Despite having some shortcomings as low individual predictive power and disability of classification of all cardiac procedures, ${ }^{6}$ it has been widely used to compare mortality among services and to evaluate the evolution of the quality of care provided. Because it is a good predictor of mortality, it has been widely used to compare mortality among services and to evaluate the evolution of the quality of care provided. ${ }^{7}$ However, the RACHS-1 score does not address individual and structural factors of a service that can directly affect surgical outcomes. ${ }^{6}$

In open heart surgery, due to cardiomyopathy pulmonary bypass ( $\mathrm{CPB})$, which has different effects on different organs of the body, it is more likely to develop complications during or after surgery. ${ }^{8}$ Almost 400 thousand open heart surgery using pump cardiovascular (CPB) are performed worldwide, out of which about $6 \%$ in children. ${ }^{9}$ To better prevent these complications and improve the prognosis of action, identification of mechanisms, incidence and risk factors play a major role. ${ }^{10}$ 
In a recent single-centered study with a total of 2,308 paediatric patients submitted to cardiac surgery with cardiopulmonary bypass support, Xien Zeng et al., ${ }^{11}$ noted that 677 (29.3\%) of the surgeries resulted in postoperative complications and 1.631 (70.7\%) did not. The mean surgical age was $22( \pm 30)$ months, and $1,151(49.9 \%)$ patients were male. The risk factors evaluated for 2,308 patients stratified by postoperative complication revealed that patients who underwent surgery and experienced postoperative complications were significantly younger, lighter and shorter. Lower blood oxygen saturation levels before and after surgery were also associated with postoperative complication. Moreover, a longer surgical time, CPB, aortic crossclamping time and particularly delayed sternal closure were associated with complications. Therefore, the right incision thoracotomy will definitely reduce the risk of complications compared to the median sternal incision. Importantly, an emergent operation was a risk factor for complications. Certainly, patients with multiple heart defects who undergo multiple procedures in the same visit will have a higher risk of complications. ${ }^{11}$ Cavalcante CT et al., ${ }^{12}$ carried out a retrospective analysis of 3,071 patients, from January 2003 to December 2014, and noted that mortality also varied during the twelve years of records, with significant decrease despite an increase in the number of procedures, ranging from $13.3 \%$ (171/1288) to $10.4 \%(191 / 1889)$ in the period II $(P=0.014)$. Mortality in the last three years was 9\% (2012-2014). When they evaluated the deaths according to RACHS-1 category, they found that the more complex the procedure, the higher the mortality rate is $(P=0.0001)$, however when analyzing the association between RACHS-1 score and mortality in the two periods separately, was noted a decrease in mortality category in recent years, with the exception of category $6 .{ }^{12}$

\section{References}

1. Pinto Jr VC, Branco KMP, Castello RC, Carvalho W, Lima JR, Freitas SM, et al. Epidemiology of congenital heart disease in Brazil Approximation of the official Brazilian data with the literature. Rev Bras Cir Cardiovasc.2015;30(2):219-24.

2. Guimaraes JR, Guimaraes ICB. Clinical and epidemiological profiles of patients admitted to a pediatric cardiac intensive care unit. Int J Cardiovasc Sci. 2020; 33(4):331-336.

3. Roger VL. American Heart Association. Statistics Committee and Stroke Statistics Subcommittee. Heart disease and stroke statistics-2011 update: a report from the AHA. Circulation.2012;125(1):e2-e220.

4. Dolk H, Loane M, Garne E. Congenital heart defects in Europe. Circulation. 2011;123(8):841-9.
Another study analyzed a total of 325 patients: 271 with cardiopulmonary bypass and 54 without cardiopulmonary bypass. Of the 325 patients, 141 (43\%) had complications (95\% confidence interval, 38\%-49\%). Of the 325 patients, 82 (25\%) developed cardiac and $120(37 \%)$ developed extracardiac complications. The evidence from logistic regression analysis was insufficient to suggest a relationship between CPB support and the incidence of cardiac or extracardiac complications after adjusting for age, gender, previous sternotomy, and RACHS-1 levels. For patients receiving CPB, longer CPB times, higher RACHS-1 levels, and a lower temperature with $\mathrm{CPB}$ were associated with a greater number of cardiac complications. ${ }^{13}$

A reduction in postoperative complications to improve outcomes in both adults and children undergoing a variety of surgical procedures has been a general focus for many researchers. In this study cohort, the overall mortality rate of patients with complications was 5.5\% and the corresponding value in all patients was $1.6 \%$. Furthermore, the postoperative length of hospital stay, length of cardiac intensive care unit stay and mechanical ventilation duration were significantly longer for patients who experienced postoperative complications, compared to patients without complications. The ability to predict complications prior to it will really help clinicians improve the care process by optimizing critical care resources for high-risk patients. ${ }^{11}$

Identifying and correcting complications early might change the relationship of complication development and mortality; thus, the downstream effects of a given complication might differ from institution to the next, depending on the infrastructure. The associations between complications and outcome might be most important in establishing patterns to target early recognition and preventive treatment.

5. Baspinar O, Karaaslan S, Oran B, Baysal T, Elmaci AM, Yorulmaz EAM, et al. Prevalence and distribution of children with congenital heart diseases in the central Anatolian region, Turkey. Turk J Pediatr. 2006;48(3):237-43.

6. Jenkins KJ, Gauvreau K. Center-specific differences in mortality: preliminary analyses using the Risk Adjustment in Congenital Heart Surgery (RACHS-1) method. J Thorac Cardiovasc Surg. 2002;124(1):97-104.

7. Nakayama Y, Shibasaki M, Shime N, Nakajima Y, Mizobe T, Sawa T. The RACHS-1 risk category can be a predictor of perioperative recovery in Asian pediatric cardiac surgery patients. J Anesth. 2013;27(6):850-4.

8. Behrmans RE, Kleigman RM. Nelson Textbook of Pediatrics. $17^{\text {th }}$.ed Philadelphia, PA: WB Saunders Co; 2004.chap: 417,418. 
9. Hill AG, groom RC, Bechara F. Pediatric Perfusion Survey II: Expanded multivariate data analysis. In: Proceeding of the American Academy of Cardiovascular Perfusion, 12, California; 1990. p.96

10. Mirzaei M, Mirzaei S, Sepahvand E, Rahmanian Koshkaki A, Kargar Jahromi M. Evaluation of Complications of Heart Surgery in Children With Congenital Heart Disease at Dena Hospital of Shiraz. Glob J Health Sci. 2015 Aug 23;8(5):33-8.

11. Zeng X, An J, Lin R, Dong C, Zheng A, Li J, et al. Prediction of complications after pediatric cardiac surgery. Eur J Cardiothorac Surg. 2000;57(2):350-8.
12. Cavalcante CT, de Souza NMG, Pinto Jr VC, Branco KM, Pompeu RG, Teles AC, et al. Analysis of Surgical Mortality for Congenital Heart Defects Using RACHS-1 Risk Score in a Brazilian Single Center. Braz J Cardiovasc Surg. 2016;31(3):219-25.

13. Agarwal S.H, MBBS, FAAP, Karen B. Wolfram, RN, Benjamin R. Saville, Brian S. Donahue, David P. Bichell. Postoperative complications and association with outcomes in pediatric cardiac surgery. J Thorac Cardiovasc Surg. 2014;148(2):609-16. 